\title{
Local-Field Effects in the Zero-Phonon Spectral Lines of Single Impurity Molecules in Solid Matrices at Low Temperatures
}

\author{
T.A. Anikushina ${ }^{1,2 a}$, M.G. Gladush ${ }^{2}$, A.A. Gorshelev², and A.V. Naumov ${ }^{1,2}$ \\ ${ }^{1}$ Moscow Pedagogical State University, 29 Malaya Pirigivskaya Str. Moscow 119991, Russia \\ ${ }^{2}$ Institute for Spectroscopy RAS, 5 Fizicheskaya St.r, Troitsk, Moscow 142190, Russia
}

\begin{abstract}
In this work, we study the relation between the widths of the zero-phonon lines (ZPL) of single impurity molecules (SM) in solid matrices with local effective values of the refractive index $\mathrm{n}$. Since at ultralow temperatures the width of SM ZPL is determined mainly by the radiative lifetime $T_{1}$, while the local-field effects produce the function $T_{1}(n)$, SM spectroscopy makes it possible to measure the distribution of $n$ local values in solids. The approach proposed here for studying local fluctuations of $n$ has been tested on the example of doped polymers and molecular crystals.
\end{abstract}

Keywords: single-molecule, super-resolution, local-field, zero-phonon line, refractive index, fluctuations, polymer, molecular crystal

Most problems in quantum optics involve consideration of nanoscale (nanostructured) materials and/or single quantum emitters. In this context, it becomes of critical importance to know about fluctuations of various characteristics of the material on the nanometer scale and the effect of the local environment on the photophysical characteristics of quantum emitters. A significant amount of information in this area comes from cryogenic fluorescence microscopy and spectroscopy of single chromophore molecules embedded in transparent (in the visible range of wavelengths) solid media [1-3].

The essence of these methods is that they allow to detect zero-phonon lines (ZPL), which corresponds to electronic transitions in impurity molecules. ZPL parameters (frequency, intensity, width, and dynamics) are very sensitive to the local environment of the corresponding chromophore SM. This fact makes SMs good candidates for spectral probes to obtain data on the structure and the internal dynamics of solids. In present study, we demonstrate a method for probing of the local fluctuations of refractive index $n$ in solids by the analysis of zero-phonon spectral lines (ZPL) of single dye molecules at ultra-low temperatures. [4]

The temperature $T$ dependence of the homogeneous spectral width of ZPL is determined by three main contributions, i.e.

$$
\Gamma_{Z P L}(T)=\Gamma_{0}+\Gamma_{e-t u n n}\left(T, t_{m}\right)+\Gamma_{e-p h o n}(T),
$$

where $\Gamma_{0}=1 / 2 \pi T_{1}$ is ZPL width, determined by the excited state natural lifetime $T_{1} ; \Gamma_{e-t u n n}\left(T, t_{m}\right)$, $\Gamma_{e-p h o n}(T)$ - are the contributions to ZPL broadening produced by the interaction of the electron transition in SM with the elementary excitations in the matrix of tunneling and vibrational types respectively. At

${ }^{\text {a }}$ Corresponding author: anikushina@1ist.ru 
$\mathrm{T}<1 \mathrm{~K}$ the contribution of vibrational and tunneling excitations is negligible and out of saturation effects due to laser excitation ZPL spectral width is limited by excited state natural lifetime $T_{1}$. Numerous studies [5] show that manifestation of the local-field effects makes $T_{1}$ a function of $n$ :

$$
T_{1}(n)=\tau_{0} / n f(n),
$$

where $f(n)$ reflects the local-field contributions and depends on the theoretical model.

Analysis of this dependence has allowed us to choose the most suitable model $f(n)=\left(\left(n^{2}+2\right) / 3\right)^{2}$ and find the "vacuum" value of the excited state lifetime $\left(\tau_{0}=12.1 \mathrm{~ns}\right)$.

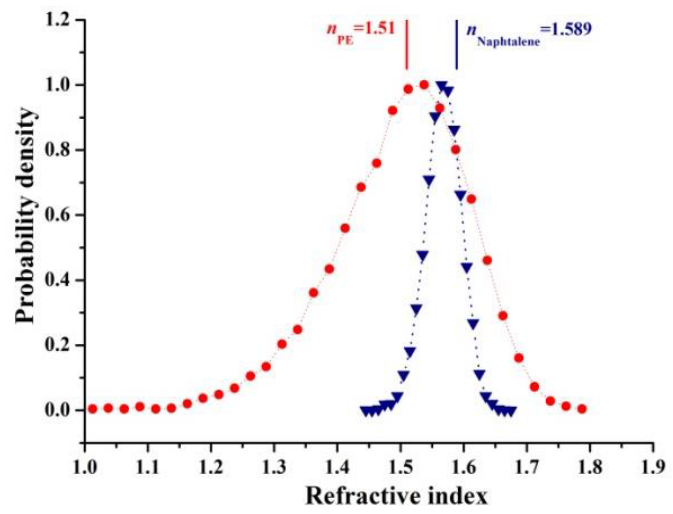

Figure 1. The distribution of the local values of the refractive index in amorphous polyethylene (red circles) and polycrystalline naphthalene (blue triangles).

In order to find the distribution of $n$ local values in real Tr-doped solids we have taken the unique experimental data on SM ZPL width distributions in amorphous polyethylene and polycrystalline naphthalene as measured at milliKelvin temperatures for further analysis [6, 7]. These distributions $\mathrm{P}_{\Gamma 0}\left(\Gamma_{0}\right)$ can be recalculated into the distributions of $P_{T 1}\left(T_{1}\right)$ and then into the distributions of refraction indices $P_{n}(n)$ by numerical solving of the corresponding Eq.(2) (Fig. 1).

Thus, the local refractive index fluctuations in solids can be found by detecting SM ZPLs at ultralow temperatures. Our analysis of the experimental data shows that there are fluctuations of $n$ in real samples. These fluctuations are more significant for more disordered materials. The maximum of the distribution $P(n)$ corresponds to the average value of $n$, obtained by traditional methods.

We show that such analysis is even more powerful, when we use sequential-parallel detection of ZPL and luminescence images of a myriad SMs [8,9] with 3D coordinates reconstruction [10].

The support from Russian Foundation for Basic Research is acknowledged (project 14-29-07270 developing of the methods for nanodiagnostics of solids), and from Russian Science Foundation (14-1201415 - statistical analysis of SMS-data).

\section{References}

1. T. Basché, W.E. Moerner, M. Orrit, U.P. Wild, Single-molecule optical detection, imaging and spectroscopy (Weinheim ; Cambridge: VCH, xiv, 250 p., 1997)

2. M. Orrit, W.E. Moerner, High Resolution Single-Molecule Spectroscopy in Condensed Matter, Physics and Chemistry at Low Temperatures (L. Khriachtchev, ed.) (Pan Stanford Publishing, Singapore, p. 381-417, 2011)

3. A.V. Naumov, Physics Uspekhi. 56. P.605-622 (2014)

4. T.A. Anikushina, M.G. Gladush, A.A. Gorshelev, A.V. Naumov, Faraday Discuss. DOI: 10.1039/C5FD00086F (2015)

5. D. V. Kuznetsov, V. K. Roerich, M. G. Gladush, J. Exp. Theor. Phys. 113. P.647-658 (2011)

6. E. A. Donley, V. Burzomato, U. P. Wild, T. Plakhotnik, J. Lumines. 8384. P. 255-259 (1999)

7. E. A. Donley, S. Bonsma, et. al., J. Lumines. 8789. P. 109-114 (2000)

8. A.V.Naumov, A.A.Gorshelev, et. al., Angewandte Chemie 48. P. 9747 - 9750 (2009)

9. A.V.Naumov, A.A.Gorshelev, et.al., Phys.Chem.Chem.Phys. 13. P. 1734 - 1742 (2011)

10. A.V. Naumov, I.Yu. Eremchev, A.A. Gorshelev, Eur. Phys. J. D. 68. P. 348 (2014) 